\title{
2-Edge-Colored Chromatic Number of Grids is at Most 9
}

\author{
Janusz Dybizbański ${ }^{1}$
}

Received: 1 September 2019/Revised: 12 February 2020/Published online: 11 March 2020

(C) The Author(s) 2020

\begin{abstract}
A 2-edge-colored graph is a pair $(G, \sigma)$ where $G$ is a graph, and $\sigma: E(G) \rightarrow$ $\{$ ',+ ,' - ' $\}$ is a function which marks all edges with signs. A 2-edge-colored coloring of the 2-edge-colored graph $(G, \sigma)$ is a homomorphism into a 2-edgecolored graph $(H, \delta)$. The 2-edge-colored chromatic number of the 2-edge-colored graph $(G, \sigma)$ is the minimum order of $H$. In this paper we show that for every 2-dimensional grid $(G, \sigma)$ there exists a homomorphism from $(G, \sigma)$ into the 2-edgecolored Paley graph $S P_{9}$. Hence, the 2-edge-colored chromatic number of the 2-edge-colored grids is at most 9. This improves the upper bound on this number obtained recently by Bensmail. Additionally, we show that 2-edge-colored chromatic number of the 2-edge-colored grids with 3 columns is at most 8 .
\end{abstract}

Keywords 2-Edge-colored coloring · Grids · Paley graphs

Mathematics Subject Classification 05C15

\section{Introduction}

In the whole paper we will use standard graph theory notations. A 2-edge-colored graph is a pair $(G, \sigma)$ where $G$ is a undirected graph and $\sigma: E(G) \rightarrow\{$ ' + ', ' - ' $\}$. For the vertex $v \in V(G)$ by $N^{-}(v)$ (resp. $N^{+}(v)$ ) we denote the set of neighbors of $v$ such that the edge to $v$ has assignment '-' (resp. ' + '). Similarly for a set $S \subset V(G)$, we define $N^{-}(S)=\bigcup_{v \in S} N^{-}(v)$ and $N^{+}(S)=\bigcup_{v \in S} N^{+}(v)$.

A 2-edge-colored coloring of a 2-edge-colored graph $G$ is a proper coloring $\phi$ of $V(G)$ such that if there exist two edges $\{u, v\}$ and $\{x, y\}$ with $\phi(u)=\phi(x)$ and $\phi(v)=\phi(y)$, then these two edges have the same sign. The 2-edge-colored

Janusz Dybizbański

jdybiz@inf.ug.edu.pl

1 Institute of Informatics, Faculty of Mathematics, Physics and Informatics, University of

Gdańsk, 80-308 Gdańsk, Poland 
chromatic number of the 2-edge-colored graph $G$, denoted by $\chi_{2}(G)$, is the minimum number of colors needed for a 2-edge-colored coloring.

Equivalently, the 2-edge-colored chromatic number $\chi_{2}(G, \sigma)$ of the 2-edgecolored graph $(G, \sigma)$ is the minimum order of the graph $(H, \lambda)$ such that $(G, \sigma)$ admits a 2-edge-colored homomorphism (preserving edges and signs) to $(H, \lambda)$. Graph $(H, \lambda)$ we call a target graph or coloring graph. The 2-edge-colored chromatic number $\chi_{2}(G)$ of a graph $G$ is defined as maximum over all 2-edgecolored of $\mathrm{G}$. For a graph class $\mathcal{F}$, we define the 2-edge-colored chromatic number $\chi_{2}(\mathcal{F})$ as the maximum over 2-edge-colored chromatic number for any members of $\mathcal{F}$. For convenience, in the rest of the paper we will omit the $\sigma$ and simply write $G$ for a 2-edge-colored graph $(G, \sigma)$ if there is no ambiguity.

In this paper we focus on 2-edge-colored chromatic number for class of 2-dimensional grids $\mathcal{G}$. The grid is defined as the graph being the Cartesian product of two paths. Similarly, by $\mathcal{G}_{k}$ we denoted the grid with $k$ columns.

The $\chi_{2}(\mathcal{G})$ was investigated in 2016 by Bensmail [1], who showed that $7 \leq \chi_{2}(\mathcal{G}) \leq 12$. Recently, in 2019, the same author [2] improved both bounds by showing, that $8 \leq \chi_{2}(\mathcal{G}) \leq 11$, determine the exact value $\chi_{2}\left(\mathcal{G}_{2}\right)=5$ and bounds $7 \leq \chi_{2}\left(\mathcal{G}_{3}\right) \leq 9$.

In Sect. 2 we define 2-edge-colored Paley graph $S P_{q}$ and focus on $S P_{9}$ as a target graph in 2-edge-colored homomorphisms. That graph was used in this context earlier, for example Montejano et al. [6] show that there exist 2-edge-colored homomorphism from every 2-edge-colored outerplanar graph to $S P_{9}$. Some other application of that target graph we can find in [7].

In Sect. 3 we prove:

Theorem 1 For every 2-edge-colored grid $G$ there exists a 2-edge-colored homomorphism $h: G \rightarrow S P_{9}$.

Corollary $2 \quad 8 \leq \chi_{2}(\mathcal{G}) \leq 9$.

In Sect. 4 we focus on grids with 3 columns and improve upper bound given by Bensmail. More precisely we show that:

Theorem 3 For every 2-edge-colored grid $G$ with 3 columns there exists a 2-edgecolored homomorphism $h: G \rightarrow S P_{9} \backslash\{0\}$.

Corollary $4 \quad 7 \leq \chi_{2}\left(\mathcal{G}_{3}\right) \leq 8$.

\section{Graph $S P_{9}$}

Let $q$ be a prime power such that $q \equiv 1(\bmod 4)$ and $\mathbb{F}_{q}$ be finite field of order $q$. The Paley graph $P_{q}$ is undirected graph with vertex set $V\left(P_{q}\right)=\mathbb{F}_{q}$ and edge set $E\left(P_{q}\right)=\left\{\{x, y\}: y-x\right.$ is a non-zero square in $\left.\mathbb{F}_{q}\right\}$. Notice that -1 is a square in $\mathbb{F}_{q}$. Hence, if $x-y$ is a square, then $y-x$ is a square.

The 2-edge-colored Paley graph $S P_{q}$ is 2-edge-colored graph $\left(K_{q}, \sigma\right)$, where $K_{q}$ is the complete graph on vertices $\mathbb{F}_{q}$ and $\sigma(\{x, y\})=^{\prime}+^{\prime} \Longleftrightarrow y-x$ is a non-zero square in $\mathbb{F}_{q}$. In this paper we use 2-edge-colored Paley graph $S P_{9}$ build over the 
Galois' field $G F\left(3^{2}\right)$. Elements of this field are the polynomials of degree 1 over $G F(3)$ with multiplication modulo $x^{2}+1$. Elements $\{0,1,2, x, 2 x\}$ are squares and $\{x+1, x+2,2 x+1,2 x+2\}$ are non squares. The graph $P_{9}$ is presented on Fig. 1 .

Lemma 5 [8] $S P_{q}$ is isomorphic to the 2-edge-colored graph constructed by flipping signs of all edges.

The following properties of $S P_{9}$ are well know:

\section{Lemma 6}

1. The Paley graph $\mathrm{SP}_{9}$ has 72 automorphisms.

2. For every non-zero square $a \in \mathbb{F}_{9}$ and every $b \in \mathbb{F}_{9}$, the function $h_{a, b}(y)=$ $a y+b$ is an automorphism in $S P_{9}$.

3. The reflection function $r: S P_{9} \rightarrow S P_{9}$, which maps the polynomial $u x+v$ to $v x+u$ is an automorphism in $S P_{9}$.

4. Each automorphism $h$ in $S_{9}$ is either of the form $h=h_{a, b}$ or is a composition $h=r \circ h_{a, b}$ for some $a$ and $b$.

Lemma 7 The Paley graph $\mathrm{SP}_{9}$ is vertex-transitive and edge-transitive. Moreover for every two induced subgraphs $S_{1}$ and $S_{2}$ with $\left|S_{1}\right|=\left|S_{2}\right|=3$ and the same number of edges marked with'-', there is an automorphism of $S P_{9}$ which maps $S_{1}$ on $S_{2}$.

Lemma 8 For every vertex $v \in V\left(S P_{9}\right),\left|N^{+}(v)\right|=4$ and $\left|N^{-}(v)\right|=4$.

We shall call 3-elements induced subgraph $T \subset V\left(S P_{9}\right)$ a triangle if all its edges are marked with the same sign. Furthermore, we shall say that set $S \subset V\left(S P_{9}\right)$ is triangle free if the subgraph of $S P_{9}$ induced by $S$ does not contain a triangle.

Lemma 9 For every vertex $v \in V\left(S P_{9}\right)$, the sets $N^{+}(v)$ and $N^{-}(v)$ are triangle free.

Proof By Lemmas 5 and 7, it is enough to prove this lemma for vertex $v=0$ and $N^{+}(v)$. The subgraph of $S P_{9}$ induced by $N^{+}(0)=\{1,2, x, 2 x\}$ contains two disjoint edges signed ' + ' $(\{1,2\}$ and $\{x, 2 x\})$ and the cycle $C_{4}$ signed '-' $(1,2 x, 2, x)$.

Fig. 1 Paley graph $S P_{9}$. Solid lines represent edges signed by '+'

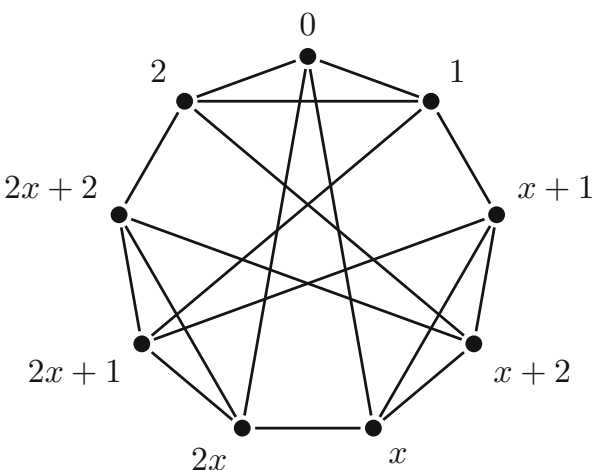


Lemma 10 Suppose that $S \subset V\left(S P_{9}\right),|S|=3$ and $S$ is triangle free, then $\left|N^{+}(S)\right|=$ 8 and $\left|N^{-}(S)\right|=8$.

Proof By Lemma 5 and 7, it is sufficient to consider the triangle free set $S=\{0,1, x\}$. Then $N^{+}(S)=S P_{9} \backslash\{2 x+2\}$ and $N^{-}(S)=S P_{9} \backslash\{0\}$.

\section{Proof of Theorem 1}

Consider a path $(u, v, w)$ with arbitrary signs on the edges $\{u, v\}$ and $\{v, w\}$. Suppose that we have: an arbitrary 3-elements triangle free set $S_{1} \subset V\left(S P_{9}\right)$ of colors available in $u$ and an arbitrary color $b \in V\left(S P_{9}\right)$ for the vertex $w$. Then there is a 3-elements triangle free set $S_{2} \subset V\left(S P_{9}\right)$ available in $v$. More precisely:

Lemma 11 Consider a path $(u, v, w)$ with arbitrary signs on the edges $\{u, v\}$ and $\{v, w\}$. For every 3-elements triangle free set $S_{1} \subset V\left(S P_{9}\right)$ and every color $b \in S P_{9}$, there exists a 3-elements triangle free set $S_{2} \subset V\left(S P_{9}\right)$ such that for each $s_{2} \in S_{2}$ there exists $s_{1} \in S_{1}$ and a coloring $c:(u, v, w) \rightarrow V\left(S P_{9}\right)$ with $c(u)=s_{1}, c(v)=s_{2}$, $c(w)=b$.

Proof We will prove the lemma in case when both edges of path $(u, v, w)$ are marked with ' + '. In any other case the proof is similar..

By Lemmas 10 and $8,\left|N^{+}\left(S_{1}\right)\right|=8$ and $\left|N^{+}(b)\right|=4$. Hence, there exists 3-elements set $S_{2} \subset N^{+}\left(s_{1}\right) \cap N^{+}(b)$. By Lemma 9, the set $S_{2}$ is triangle free.

Proof of Theorem 1 We color the 2-edge-colored grid $G$ row by row. It is easy to color first row by $\mathrm{SP}_{9}$ (in fact, we can do it using only four colors, for example by $\left.N^{+}(0)=\{1,2,2 x, 2 x+1\}\right)$. Assume now that, for $k>1$, the first $k-1$ rows of $G$ have been already colored and we color $k$-th row.

Let us denote the vertices in the $k-1$-th row by $a_{1}, a_{2}, \ldots, a_{n}$ and the vertices in the $k$-th row by $b_{1}, b_{2}, \ldots, b_{n}$. By Lemma 8 , the vertex $b_{1}$ can be colored by four possible colors. By Lemma 9, any three of these colors form a triangle free set. Let us denote by $S_{1}$ any of these sets. Now for each $i=2,3, \ldots, n$ we define set $S_{i}$ as a result of applying Lemma 11 for the set $S_{i-1}$ and the color $h\left(a_{i}\right)$.

Now we can color vertices $b_{1}, b_{2}, \ldots, b_{n}$ in reverse order. First we choose any color in $S_{n}$ for $h\left(b_{n}\right)$. For $h\left(b_{n-1}\right)$ we set the color in $S_{n-1}$ such that the sign of the edge $\left(h\left(b_{n-1}\right), h\left(b_{n}\right)\right)$ in $S P_{9}$ equals to the sign of the edge $\left(b_{n-1}, b_{n}\right)$ in the grid $G$. Notice that for each $s \in S_{n-1}$, the sign of the edge $\left(a_{n-1}, b_{n-1}\right)$ in the grid is equals to the sign of the edge $\left(s, h\left(a_{n-1}\right)\right)$ in $S P_{9}$. Consecutive vertices we color in the same way.

\section{Grids with 3 Columns}

In the previous section, we show that every 2-edge-colored grid admits 2-edgecolored coloring with 9 colors. This bound can be improved when we consider grids with a small number of columns. Bensmail [2] shows that 2-edge-colored chromatic number of grids with two columns is equal to 5 . From the same paper we know that 
$7 \leq \chi_{2}\left(\mathcal{G}_{3}\right) \leq 9$. We can improve this upper bound by showing that there exists a homomorphism from every 2-edge-colored grids with 3 columns to $S P_{9}^{-0}$, see Fig. 2. $S P_{9}^{-0}$ is construct from $S P_{9}$ by removing vertex 0 . It is easy to see that this graph has 8 automorphisms. Namely, four rotations $(f(y)=a y$ where $a$ is a nonzero square) and four reflection. $S P_{9}^{-0}$ is also isomorphic to the graph constructed by flipping signs of all edges.

Lemma 12 Consider a path $(u, v, w)$ with arbitrary signs on the edges $\{u, v\}$ and $\{v, w\}$. For every color $a \in S P_{9}^{-0}$ and every $b \notin\{a,-a\}$ there exists a coloring of path $c:(u, v, w) \rightarrow V\left(S P_{9}^{-0}\right)$ such that $c(u)=a$ and $c(w)=b$.

Proof By automorphisms of $S P_{9}^{-0}$ and the fact that it is isomorphic to the graph constructed by flipping signs of all edges, we can only consider the case when $a=1$.

- $N^{+}\left(N^{+}(1)\right)=V\left(S P_{9}^{-0}\right) \backslash\{2\}$

- $N^{+}\left(N^{-}(1)\right)=V\left(S P_{9}^{-0}\right) \backslash\{1\}$

- $N^{-}\left(N^{+}(1)\right)=V\left(S P_{9}^{-0}\right) \backslash\{1\}$

- $N^{-}\left(N^{-}(1)\right)=V\left(S P_{9}^{-0}\right)$

This observation leads to partition of $S P_{9}^{-0}$ into four sets: $\{1,2\},\{x, 2 x\},\{x+$ $1,2 x+2\}$ and $\{x+2,2 x+1\}$. Regardless of the signs of the edges of the path $(u, v, w)$, if endpoints are colored using colors from two different sets, then we can color the middle point of the path.

Proof of Theorem 3 Let us consider the partition of $V\left(S P_{9}^{-0}\right)$ into four sets: $\{1,2\}$, $\{x, 2 x\},\{x+1,2 x+2\}$ and $\{x+2,2 x+1\}$. Observe that each set contains a pair $\{a,-a\}$ and that for every vertex $v \in S P_{9}^{-0}$, both $N^{+}(v)$ and $N^{-}(v)$ contain an element in at least three sets. We color the grid row by row. Three vertices in the first row we can easily color with $S P_{9}^{-0}$. Assume now that, for $k>1$, the first $k-1$ rows of $G$ have been already colored and we color $k$-th row. Let us denote the

Fig. 2 2-edge-colored graph $S P_{9}^{-0}$. Solid lines represent edges signed by ' + '

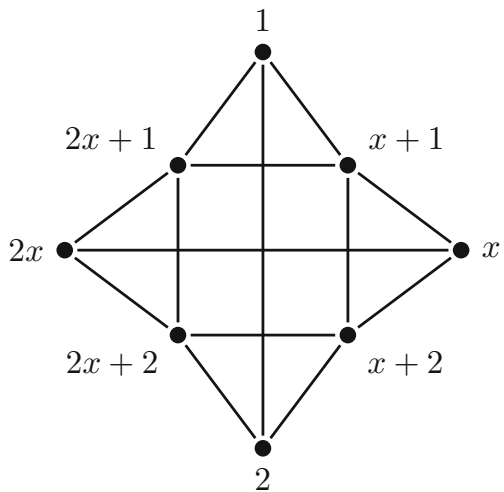


vertices in the $k-1$-th row by $a_{1}, a_{2}, a_{3}$ and the vertices in the $k$-th row by $b_{1}, b_{2}, b_{3}$. Now color vertices $b_{1}, b_{2}, b_{3}$ starting from $b_{2}$. Depending on sign of the edge $\left\{a_{2}, b_{2}\right\}$, for $c\left(b_{2}\right)$ use color in $N^{+}\left(c\left(a_{2}\right)\right)$ or $N^{-}\left(c\left(a_{2}\right)\right)$ which does not belong to the pairs: $\left\{c\left(a_{1}\right),-c\left(a_{1}\right)\right\}$ and $\left\{c\left(a_{3}\right),-c\left(a_{3}\right)\right\}$. By Lemma 12, we can extend this coloring to vertex $b_{1}$ (using path $\left(a_{1}, b_{1}, b_{2}\right)$ ) and to $b_{3}$ (using $\left(b_{2}, b_{3}, a_{3}\right)$ ).

\section{Discussion}

In this article, we show that $S P_{9}$ colors all 2-edge-colored grids and that $S P_{9}^{-0}$ colors all 2-edge-colored grids with 3 columns. Combining this with known lower bounds we have $8 \leq \chi_{2}(\mathcal{G}) \leq 9$ and $7 \leq \chi_{2}\left(\mathcal{G}_{3}\right) \leq 8$. In our opinion, $S P_{9}^{-0}$ is the best candidate to lower the upper bound for $\chi_{2}(\mathcal{G})$. However, we do not know whether $S P_{9}^{-0}$ colors all grids, and even whether it colors all grids with 4 columns. On the other hand, to increase lower bound for $\chi_{2}(\mathcal{G})$ we have to prove that there exists a 2edge-colored grid $G$ such that there is no homomorphism $G \rightarrow S P_{9}^{-0}$. Finding such a grid also seems to be difficult.

Many authors investigate the relations between 2-edge-coloring and oriented coloring. Oriented coloring (see [9] for a short survey) of an oriented graph $G$ is a homomorphism from $G$ to a tournament $H$. The oriented chromatic number $\chi_{o}(G)$ for oriented graph and for undirected graph are define analogously to 2-edgecolored case. A lot of research on 2-edge-colored and oriented coloring used the same techniques and get similar results. For example, in both cases, authors often use Paley graphs (or Paley tournaments) for the target graph. These may result from the similarity of the definitions: in both cases, edges are in one of two states (one of two signs or one of two directions). Despite these similarities, it is known that $\chi_{o}(G)-\chi_{2}(G)$ and $\chi_{2}(G)-\chi_{o}(G)$ can be arbitrarily large [3].

For grids, these two colorings seems to behave very similar. For example, for grids with 2 columns, we know that: $\chi_{2}\left(\mathcal{G}_{2}\right)=5$ [2] and $\chi_{o}\left(\mathcal{G}_{2}\right)=6$ [5]. For grids with 3 columns, we have $7 \leq \chi_{2}\left(\mathcal{G}_{3}\right) \leq 8$ and $\chi_{o}\left(\mathcal{G}_{3}\right)=7$ [10]. The difference between these two chromatic number is very small. Therefore one can hope that also for the class of grids, they are close to each other. We know that oriented chromatic number for grids lies between 8 and $11[4,5]$. Upper bound has not been improved since the first paper on the topic.

Open Access This article is licensed under a Creative Commons Attribution 4.0 International License, which permits use, sharing, adaptation, distribution and reproduction in any medium or format, as long as you give appropriate credit to the original author(s) and the source, provide a link to the Creative Commons licence, and indicate if changes were made. The images or other third party material in this article are included in the article's Creative Commons licence, unless indicated otherwise in a credit line to the material. If material is not included in the article's Creative Commons licence and your intended use is not permitted by statutory regulation or exceeds the permitted use, you will need to obtain permission directly from the copyright holder. To view a copy of this licence, visit http:// creativecommons.org/licenses/by/4.0/. 


\section{References}

1. Bensmail, J.: On the signed chromatic number of grids (2016). hal-01349656

2. Bensmail, J.: On the 2-edge-coloured chromatic number of grids. Aust. J. Combin. 75(3), 365-384 (2019)

3. Bensmail, J., Duffy, C., Sen, S.: Analogues of cliques for (m, n)-colored mixed graphs. Graph. Combin. 33(4), 735-750 (2017)

4. Dybizbański, J., Nenca, A.: Oriented chromatic number of grids is greater than 7. Inf. Process. Lett. 112(4), 113-117 (2012)

5. Fertin, G., Raspaud, A., Roychowdhury, A.: On the oriented chromatic number of grids. Inf. Process. Lett. 85(5), 261-266 (2003)

6. Montejano, A., Ochem, P., Pinlou, A., Raspaud, A., Sopena, É.: Homomorphisms of 2-edge-colored graphs. Discr. Appl. Math. 158(12), 1365-1379 (2010)

7. Ochem, P., Pinlou, A., Sen, S.: Homomorphisms of 2-edge-colored triangle-free planar graphs. J. Graph Theory 85(1), 258-277 (2017)

8. Sachs, H.: Über selbstkomplementäre graphen. Publ. Math. Debrecen 9, 270-288 (1962)

9. Sopena, É.: Homomorphisms and colourings of oriented graphs: an updated survey. Discr. Math. 339, 1993-2005 (2016)

10. Szepietowski, A., Targan, M.: A note on the oriented chromatic number of grids. Inf. Process. Lett. 92(2), 65-70 (2004)

Publisher's Note Springer Nature remains neutral with regard to jurisdictional claims in published maps and institutional affiliations. 